\title{
Temperature and concentration dependent viscosity and gelation temperature of ABA triblock copolymer solutions
}

\author{
K. Visscher and P.F. Mijnlieff
}

Rheology Group, Dept. of Applied Physics, Twente University, P.O. Box 217, 7500 AE Enschede, The Netherlands

\begin{abstract}
In solutions of ABA-triblock copolymers in a poor solvent for $A$ thermoreversible gelation can occur. A three-dimensional dynamic network may form and, given the polymer and the solvent, its structure will depend on temperature and polymer mass fraction. The zero-shear rate viscosity of solutions of the triblock-copolymer polystyrene-polyisoprene-polystyrene in n-tetradecane was measured as a function of temperature and polymer mass fraction, and analyzed; the polystyrene blocks contained about 100 monomers, the polyisoprene blocks about 2000 monomers. Empirically, in the viscosity at constant mass fraction plotted versus inverse temperature, two contributions could be discerned; one contribution dominating at high and the other one dominating at low temperatures. In a comparison with theory, the contribution dominating at low temperatures was identified with the Lodge transient network viscosity; some questions remain to be answered, however. An earlier proposal for defining the gelation temperature $T_{\text {gel }}$ is specified for the systems considered, and leads to a gelation curve; $T_{\text {gel }}$ as a function of polymer mass fraction.
\end{abstract}

Key words: Triblock-copolymer solution; zero shear-rate viscosity; dynamic network; thermo-reversible gelation; gelation temperature

\section{List of symbols}

\section{Mathematical symbols}

(1) functional dependence; e.g., $f\{x\}$ means $f$ is a function of $x$

${ }^{p} \log \operatorname{logarithm}$ to the base number $p$; e.g. ${ }^{10} \log$ is the common logarithm

exp exponential function with base number $e$

sin trigonometric sine function

lim limit operation

- in integral sign:

Cauchy Principal Value of integral,

e.g., $f_{0}^{\infty} d x f\{x\}$

$\frac{d}{d x} \quad$ derivative to $x$

$\frac{\partial}{\partial x}$ partial derivative to $x$

\section{Latin symbols}

$\bar{a} \quad$ dimensionless constant constant with dimension of absolute temperature constant with dimension of absolute temperature dimensionless constant mass fraction dimensionless constant constant with dimension of absolute temperature dimensionless constant

constant with dimension of absolute temperature

base number of natural (or Naperian) logarithm distribution function of inverse relaxation times $\lambda$ relaxation strength relaxation function distribution function of relaxation times $\tau$ reaction constant enthalpy of a molecule

$H \quad$ Heaviside unit step function $i \quad$ complex number defined by $i^{2}=-1$ 
$j(0)$ constant with dimension of viscosity

$j \quad$ index number

$k \quad$ Boltzmann's constant

$k_{H} \quad$ Huggins' coefficient

$m$ mass of a molecule

$n$ number

$N \quad$ number

$p \quad$ index number

$s \quad$ entropy of a molecule

$t$ time

$T$ absolute temperature

\section{Greek symbols}

$\alpha \quad$ as index: type of polymer molecule

$\beta \quad$ as index: type of polymer molecule

$\gamma \quad$ shear

as index: type of polymer molecule

$\dot{\gamma}$ shear rate

$\delta \quad$ small variation; e.g. $\delta T$ is a small variation in $T$ relative deviation

Dirac delta distribution

as index: type of polymer molecule

$\Delta$ difference; e.g. $\Delta \mu$ is a difference in chemical potential

$\zeta \quad$ constant with dimension of absolute temperature

$\eta \quad$ (complex) viscosity

$\bar{\eta} \quad$ constant with dimension of viscosity

$[\eta]$ intrinsic viscosity number

$\lambda$ inverse of relaxation time $\tau$

$\mu \quad$ chemical potential

$\pi \quad$ number $p i$; circle circumference divided by its diameter

$\varrho \quad$ mass per unit volume

$\tau \quad$ relaxation time

shear stress

$\omega$

\section{Introduction}

This paper deals with the zero shear rate viscosity of solutions of an ABA triblock copolymer $(A=$ polystyrene, about 100 monomers; $\mathrm{B}=$ polyisoprene, about 2000 monomers) in n-tetradecane. Due to the poor solubility, decreasing with decreasing temperature, of polystyrene in this solvent, the A-ends tend to associate in domains (in solutions of the $A B$ diblock copolymers micelles result). In the ABA solutions, the polymer molecules may connect domains with each other. When they do, supermolecular structures, eventually assuming macroscopic dimensions, may result.

We chose these systems as model systems for studying thermoreversible gelation. They are attractive because the mechanism of the formation of domains, which may operate as crosslinks, is qualitatively understood, and because all connections between domains are taken care of by similar chains, the B-parts of the ABA molecules.

The systems considered have much in common with the ionomer solutions (AB- und ABA-type) studied by Möller et al. [1 a]. There, too, all chains connecting domains are the same. On the other hand, the domain size (number of A-ends in a domain) in our systems might be less sharply peaked than it appears to be in the ionomer solutions.

Another important analogy is that in both systems physical networks may form. This name is reserved [1 b] for those systems in which crosslink formation is not due to covalent bonds. As a result, A-ends are not permanently part of one and the same domain, and connections between domains will form as well as disappear.

These systems are liquids in the rheological sense: after a deformation, the stress needed to maintain that deformation sooner or later becomes isotropic. Being a liquid, their steady shear viscosity is an even function of shear rate, and approaches to a positive constant, the Newtonian viscosity $\eta$, in the limit or zero shear rate.

The Newtonian viscosity is a property of a liquid system in its state of thermodynamic equilibrium and, thus, for a given system (solute and solvent), depends on pressure (which was the same in all measurements and which will no longer be mentioned), temperature and polymer mass fraction only. It does so through the way in which these quantities determine the molecular structure and its dynamics. This is reflected in the relation

$$
\eta=\sum_{j=1}^{N} G_{j} \tau_{j}
$$

between the Newtonian viscosity, and the strengths $G_{j}$ and relaxation times $\tau_{j}$ of the relaxation mechanisms, numbered $j$, of which there are a great many, in general; we come back to the background of relation (1) later on.

From the relaxation spectrum, which is the set of $\left\{G_{j}, \tau_{j}\right\}$ values, some information about the structure of a system can be derived on using molecular 
theories. More usually, the spectrum or some properties determined by it are measured in order to check assumptions about the molecular structure. Clearly, the righthand side of relation (1) is just one, very global, property of the spectrum. Nevertheless, measurements of viscosity as a function of polymer mass fraction and, in particular, of temperature will appear to allow for at least some conclusions about the contributions of the various relaxation mechanisms to the sum in (1).

Our interest in Newtonian viscosity measurements arose during an extensive investigation into the linear viscoelasticity of $\mathrm{ABA}$ solutions at various temperatures and polymer mass fractions, while using samples differing from each other in A- or B-block length. The storage and loss modulus $\left(G^{\prime}\right.$ and $\left.G^{\prime \prime}\right)$ were measured at frequencies between $2 \mathrm{mHz}$ and $2 \mathrm{~Hz}$. These measurements are reported elsewhere [2].

The moduli, as it is to be expected, increase strongly with decreasing temperature, in particular in the low-frequency region. The storage modulus displays a tendency towards developing a "plateau", and in some cases a nice plateau is observed. Such a plateau, a frequency independent region in $G^{\prime}$, is indicative for the presence of a long-range network held together by permanent or long-lasting crosslinks [3 a].

When a distinct plateau is absent, one may ask whether this is due to the time an A-end is part of one and the same domain being short, or to the dimensions of network-like structures being rather small. For deciding about the presence of a long-range network, albeit a temporary one, we could not investigate the criterion introduced by Winter and Chambon $[4,5]$ for defining the "gel-point". This criterion is the parallelity of $\log \left(G^{\prime}\right)$ and $\log \left(G^{\prime \prime}\right)$ versus $\log$ ("frequency") over some decades of frequency. We did not find a convincing parallelity, and timetemperature superposition [ $3 \mathrm{~b}]$, often used to extend the frequency range, did not work. This is not surprising in view of the influence temperature may be expected to have on the molecular structure and, therefore, on the spacing of relaxation times.

A quantity which we could not derive from the dynamic measurements, and which might shed some light on the molecular structure, was the Newtonian viscosity $\eta$ of our systems. It equals the zero-frequency limit of $G^{\prime \prime} / \omega$, where $\omega$ is angular frequency, but in most cases the $G^{\prime \prime}$ behavior did not allow for the extrapolation needed. This called for direct measurements of $\eta$. Preliminary measurements had revealed a shear-thickening followed by a shear-thinning behavior, so in these measurements a careful extrapolation towards zero shear rate was needed.
Results of such measurements are analyzed in this paper. Some insight into the influence of temperature and polymer mass fraction on the relaxation spectrum will be obtained. It will bring us to a proposal about what might be called the "gelation temperature" of the systems investigated.

\section{Measurements}

The viscosity of the system has been measured as a function of temperature and polymer mass fraction. Measurements were carried out with a Carrimed Controlled Stress Rheometer in the cone-and-plate configuration (angle between cone and plate $18 \mathrm{mRad}$, cone diameter $60 \mathrm{~mm}$ ). From the measurements, the zero shear rate viscosity $\eta$ was obtained by curve fitting to the expression

$$
\tau=\eta \dot{\gamma}+\alpha \dot{\gamma}^{3},
$$

in view of the shear stress $\tau$ being an odd function of the shear rate $\dot{\gamma}$. In (2), $\alpha$, like $\eta$, is a constant, depending on temperature and polymer mass fraction only. The existence of a non-zero term $\alpha \dot{\gamma}^{3}$ (and other nonlinear terms, if there are) may be due to the formation or break-up of molecular structures at finite shear rate. Such structure changes do not reflect themselves in the Newtonian viscosity $\eta$, this being a property of a liquid in its state of thermodynamic equilibrium.

The mass fractions $c_{\text {pol }}$ of the solutions investigated were $0.015,0.030,0.040,0.050$, and 0.060 . The temperatures imposed ranged from $293 \mathrm{~K}$ to $348 \mathrm{~K}$, with intervals of $5 \mathrm{~K}$.

An example of a curve fit according to (2) is given in Fig. 1.

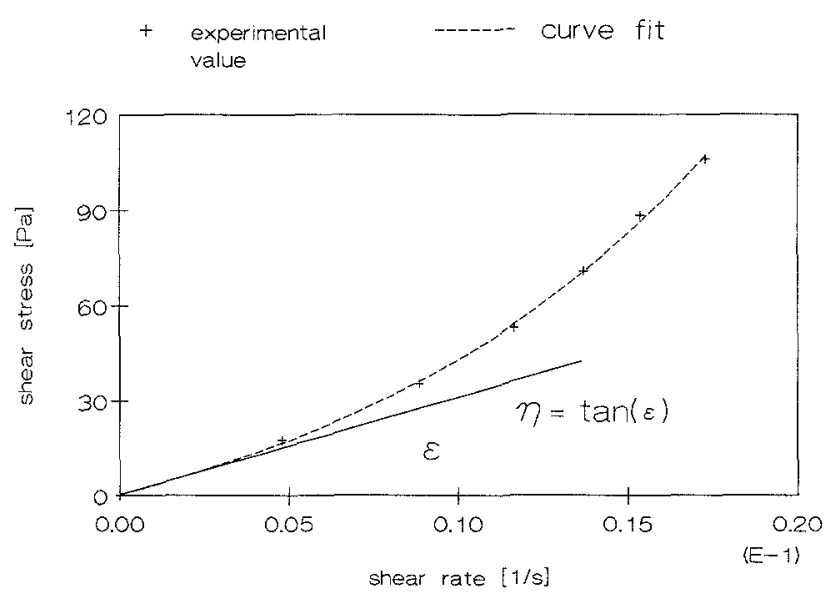

Fig. 1. Determination of the zero-shear rate viscosity, for the sample with polymer mass fraction 0.04 , at $20^{\circ} \mathrm{C}$. Curve fit according to $\mathrm{Eq}$. (2) 


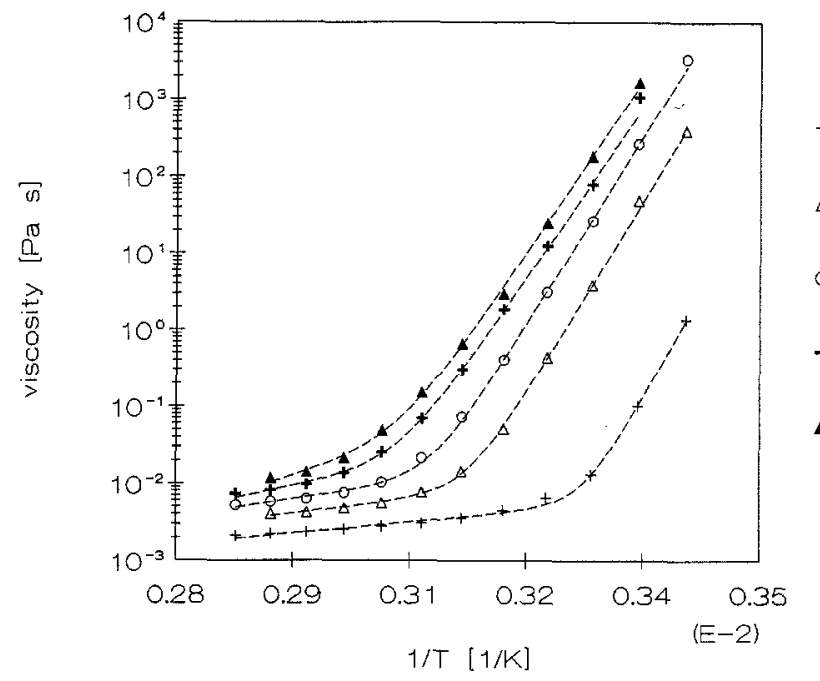

The viscosities resulting from 56 of such curve fits are given by the points in Fig. 2. The dashed curves are the fits to the relation [Eq. (55), below], at which we arrive in the section "Gelation temperature, etc.". In view of the high temperature dependence of the viscosity in the low temperature region, the main source of inaccuracy in that region will be the limitation to about $\pm 0.2^{\circ} \mathrm{C}$ accuracy in the temperature control.

For measuring relatively low viscosities, as observed in the high temperature region, the Carrimed Rheometer is not the most suitable instrument. Yet we preferred it to a Ubbelohde viscometer, in view of the non-homogeneous $\dot{\gamma}$-profile in the latter instrument.

\section{Empirical expression for the temperature and concentration dependence of the viscosity}

We now look for an expression which properly represents the viscosity behavior observed.

In Fig. 2, at any of the mass fractions considered, the viscosity curves that might be drawn by the eye through the points for one and the same mass fraction appear to consist of a low-slope part, a part in which the slope of $\log (\eta)$ vs $1 / T$ strongly increases, and a high-slope part.

Viscosities in the high-slope and in the low-slope part will be indicated by $\eta_{\text {high }}$ and $\eta_{\text {low }}$, and we first analyze the temperature and mass fraction dependence of these quantities separately.

\section{Analysis of $\eta_{\text {high }}$}

From the experimental results at "low" temperatures, Fig. 3 is obtained. It gives $\eta_{\text {high }}$ per unit of fraction

0.015

$\Delta$ fraction

0.03

- fraction

0.04

+ fraction

0.05

- fraction

0.06

Fig. 2. Zero shear rate solution viscosity as a function of inverse absolute temperature. Symbols: experimental values. Dashed curves: curve fits, at constant polymer mass fraction, to the relation (55)

polymer mass fraction, as a function of $c_{\text {pol }}$ at three (low) temperatures. It can be seen that $\eta_{\text {high }}$ at constant $T$ tends to a limiting value. When the same quantity is plotted vs inverse mass fraction, Fig. 4 results.

The plots in Fig. 4 are described by the empirical relation:

$$
\eta_{\text {high }}=c_{\text {pol }} \exp \left(\frac{-B}{c_{\text {pol }}}\right) f\{T\},
$$

where $B$ is a positive constant and $f(T)$ a function of temperature alone.

On the other hand, from Fig. 2, one finds the empirical relation:

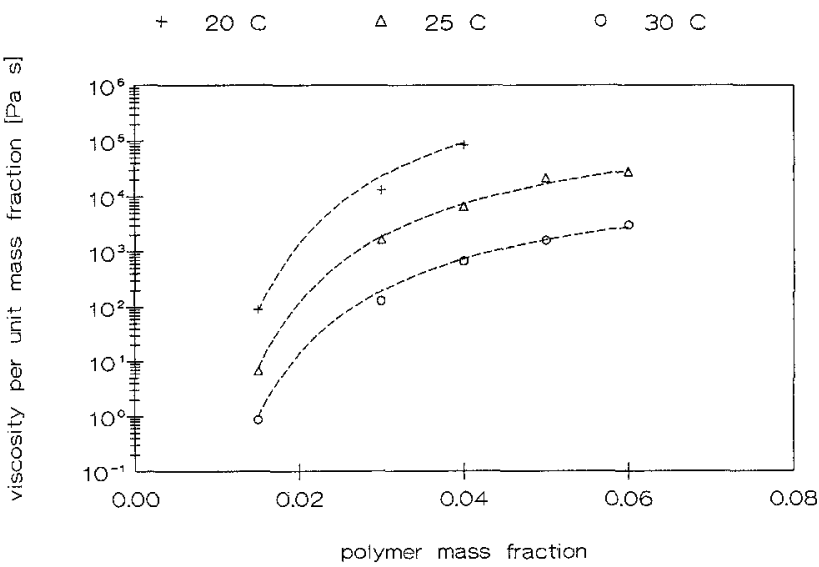

Fig. 3. Zero shear rate solution viscosity per unit polymer mass fraction, as a function of polymer mass fraction, at "low" temperatures. Symbols: experimental values. Dashed curves: curve fits corresponding to curve fits in Fig. 4 


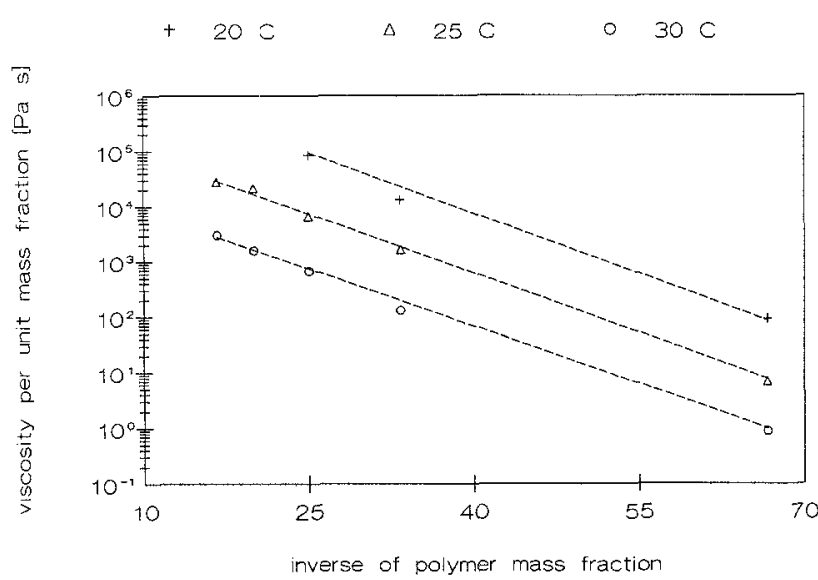

Fig. 4. Zero shear rate solution viscosity per unit polymer mass fraction, as a function of inverse polymer mass fraction, for "low" temperatures. Symbols: experimental values. Dashed curves: curve fits according to Eq. (3A)

$$
\eta_{\text {high }}=g\left\{c_{\text {pol }}\right\} \exp \left(\frac{b}{T}\right)
$$

in which $b$ is a positive constant, and $g\left\{c_{\text {pol }}\right\}$ a function of polymer mass fraction alone.

From the equivalence of these relations we find, on separating variables:

$$
\begin{aligned}
& g\left\{c_{\mathrm{pol}}\right\}=\bar{\eta} c_{\mathrm{pol}} \exp \left(\frac{-B}{c_{\mathrm{pol}}}\right) \\
& f\{T\}=\bar{\eta} \exp \left(\frac{b}{T}\right),
\end{aligned}
$$

where $\bar{\eta}$ is the separation constant. The resulting empirical relation reads:

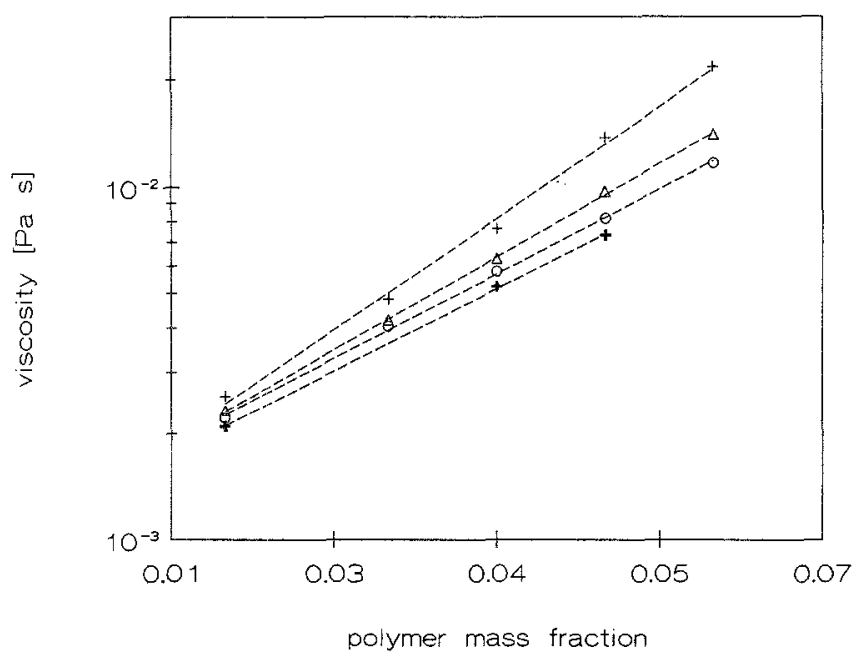

$$
\eta_{\text {high }}=\bar{\eta} c_{\mathrm{pol}} \exp \left(\frac{-B}{c_{\mathrm{pol}}}\right) \exp \left(\frac{b}{T}\right) .
$$

\section{Analysis of $\eta_{\text {low }}$}

In Fig. $5 \eta_{\text {low }}$ has been plotted logarithmically vs $c_{\text {pol }}$ at four (high) temperatures. The dashed lines in Fig. 5 suggest the following empirical relation for $\eta_{\text {low }}$ :

$$
\eta_{\text {low }}=\eta_{0}\{T\} \exp \left(d\{T\} c_{\mathrm{pol}}\right)
$$

with $d\{T\}$ a function of temperature alone, and $\eta_{0}\{T\}$ the temperature-dependent viscosity of the solvent.

On the other hand, from Fig. 2, one finds the empirical relation:

$$
\eta_{\text {low }}=j\left\{c_{\mathrm{pol}}\right\} \exp \left(\frac{D\left\{c_{\mathrm{pol}}\right\}}{T}\right)
$$

in which $j\left\{c_{\mathrm{pol}}\right\}$ and $D\left\{c_{\mathrm{pol}}\right\}$ are functions of the polymer mass fraction alone.

Combining the expressions (6) and (7), we obtain:

$$
\eta_{0}\{T\} \exp \left(d\{T\} c_{\mathrm{pol}}\right)=j\left\{c_{\mathrm{pol}}\right\} \exp \left(\frac{D\left\{c_{\mathrm{pol}}\right\}}{T}\right) \text {. }
$$

Substituting $c_{\text {pol }}=0$ yields:

$$
\eta_{0}\{T\}=j\{0\} \exp \left(\frac{D\{0\}}{T}\right),
$$

from which:

$$
\begin{aligned}
& +60^{\circ} \mathrm{C} \\
& \Delta \quad 65^{\circ} \mathrm{C} \\
& +70^{\circ} \mathrm{C} \\
& +\quad 75^{\circ} \mathrm{C}
\end{aligned}
$$

Fig. 5. Zero shear rate solution viscosity as a function of polymer mass fraction, for "high" temperatures. Symbols: experimental values. Dashed curves: curve fits according to Eq. (6) 


$$
\frac{d}{d T^{-1}} \ln \left(\eta_{0}\{T\}\right)=D\{0\}
$$

Taking the logarithm of (8) gives:

$$
\ln \left(\eta_{0}\{T\}\right)+c_{\mathrm{pol}} d\{T\}=\ln \left(j\left\{c_{\mathrm{pol}}\right\}\right)+\frac{D\left\{c_{\mathrm{pol}}\right\}}{T},
$$

from which:

$$
\frac{d}{d T^{-1}} \ln \left(\eta_{0}\{T\}\right)+c_{\mathrm{pol}} \frac{d}{d T^{-1}} d\{T\}=D\left\{c_{\mathrm{pol}}\right\} .
$$

Using (10) we find:

$$
D\{0\}+c_{\mathrm{pol}} \frac{d}{d T^{-1}} d\{T\}=D\left\{c_{\mathrm{pol}}\right\} .
$$

Separation of variables in relation (11) gives:

$$
\frac{d}{d T^{-1}} d\{T\}=c_{\mathrm{pol}}^{-1}\left(D\left\{c_{\mathrm{po}}\right\}-D\{0\}\right)=\zeta,
$$

where $\zeta$ is the separation constant.

Solution of the equation $\frac{d}{d T^{-1}} d\{T\}=\zeta$ gives:

$$
d\{T\}=d^{*}+\frac{\zeta}{T}
$$

where $d^{*}$ is an integration constant.

The final empirical relation for $\eta_{\text {low }}$ reads:

$$
\eta_{\text {low }}=\eta_{0}\{T\} \exp \left[\left(d^{*}+\frac{\zeta}{T}\right) c_{\text {pol }}\right] \text {, }
$$

with $\eta_{0}\{T\}$ given by (9).

\section{The resulting viscosity}

Having established empirical expressions for $\eta_{\text {low }}$ and $\eta_{\text {high }}$, we now look for an expression that can be fitted to all viscosities, including those in the transition zone.

As such, we try

$$
\eta=\eta_{\text {low }}+\eta_{\text {high }},
$$

on taking for $\eta_{\text {low }}$ and $\eta_{\text {high }}$ the expressions (12) and (5). That is,

$$
\begin{aligned}
\eta= & \eta_{0}\{T\} \exp \left[\left(d^{*}+\frac{\zeta}{T}\right) c_{\mathrm{pol}}\right] \\
& +\bar{\eta} c_{\mathrm{pol}} \exp \left(\frac{-B}{c_{\mathrm{pol}}}\right) \exp \left(\frac{b}{T}\right) .
\end{aligned}
$$

For $\eta_{0}\{T\}$ we used accurate data from Ubbelohde measurements carried out by P.H. J. Spitteler of this laboratory. They are well described by the expression (9), with:

$$
\begin{array}{ll}
j\{0\}=\exp (-12.2184) & {[\mathrm{Pa} \mathrm{s}]} \\
D\{0\}=1805.02 & {[\mathrm{~K}] .}
\end{array}
$$

The accuracy in $\eta_{0}\{T\}$, calculated in this way, is about $\pm 0.2 \%$ in the temperature range 283 to $323 \mathrm{~K}$. As in most more-parameter fits, in order to get an adequate curve-fit, the numbers $j\{0\}$ and $D\{0\}$ have to be given in more figures than their actual accuracy permits. This is because the uncertainties in the values of the numbers $j\{0\}$ and $D\{0\}$ are coupled. This can be seen as follows:

In a two-dimensional plot with $j\{0\}$ and $D\{0\}$ on the two axes, the line connecting points $(j\{0\}, D\{0\})$ corresponding to a certain constant r.m.s. deviation $\delta$ in general surround a region (called the $\delta$-region in this paper) around the best-fit point, say $\left(j\{0\}_{0}, D\{0\}_{0}\right)$. The accuracy range of $j\{0\}_{0}$ and $D\{0\}_{0}$ is now defined by the extreme values of $j\{0\}$ and $D\{0\}$ on the boundary of the $\delta$-region. If the r.m.s. deviation is chosen conveniently, then all points $(j\{0\}, D\{0\})$ within the $\delta$ region correspond to viscosity curves, deviating little from the best fit.

If now one just picks two values of $j\{0\}$ and $D\{0\}$, lying within their respective accuracy ranges, then the point $(j\{0\}, D\{0\})$ is not automatically within the $\delta$ region. The latter only is the case for a $\delta$-region in the shape of a rectangle with sides parallel to the axes. The conclusion is that if one chooses a value of $j\{0\}$ within the accuracy range of $j\{0\}_{0}$, then in general not all values of $D\{0\}$ within the accuracy range of $D\{0\}_{0}$ lead to acceptable viscosity curves.

With $\eta_{0}\{T\}$ given, a five-parameter fit remains. It was carried out with the Downhill Simplex Method [6]. (Details are available on request.)

The root-mean-square (r.m.s.) value of the relative deviations $\delta_{i}$, defined as

$$
\delta_{i} \equiv \frac{\eta_{i}}{\eta_{\text {measured }, i}}-1
$$


with $\eta_{i}$ calculated from (14), for the temperature and polymer mass fraction corresponding to $\eta_{\text {measured, } i}$, was taken as a measure of the quality of the fit. Minimization of this r.m.s.-value resulted in values near 0.21 , and slightly varying sets of "best" parameter values; from these we chose the following set:

$$
\begin{array}{lll}
\bar{\eta}_{\text {best }}=\exp (-120.26) & {[\mathrm{Pa} \mathrm{s}] ;} & B_{\text {best }}=0.17412 \\
b_{\text {best }}=39773 & {[\mathrm{~K}] ;} & d_{\text {best }}^{*}=-107.01 \\
\zeta_{\text {best }}=51825 & {[\mathrm{~K}] .}
\end{array}
$$

A r.m.s. relative deviation of 0.21 in the best-fit curves seems a rather high value. On a logarithmic scale (and one is forced to use it because the measured viscosities cover a large range) this is, however, not the case; an absolute deviation of ${ }^{10} \log (1.2) \simeq 0.08$ is found, while $-3<{ }^{10} \log (\eta)<4$.

Further, one could ask whether this high r.m.s. relative deviation indicates that (14) cannot match the measured viscosity data. This question can be answered by examining the influence of temperature on the data.

Because the influence of temperature on the viscosity $\eta_{\text {high }}$ is strong, and the temperature is only known with an accuracy of $\pm 0.2 \mathrm{~K}$, the accuracy of the measured viscosity data at certain fixed low temperatures is not known in detail. However, by taking partial derivatives with respect to $T$, an estimate of the error $\delta \eta$ in the viscosity data due to inaccuracies $\delta T$ in the temperature follows from the expressions (12) and (5) for $\eta_{\text {low }}$ and $\eta_{\text {high }}$ :

$$
\frac{\delta \eta_{\text {low }}}{\eta_{\text {low }}} \simeq \frac{\left(D\{0\}+\zeta c_{\text {pol }}\right)}{T} \frac{\delta T}{T} ; \quad \frac{\delta \eta_{\text {high }}}{\eta_{\text {high }}} \simeq \frac{b}{T} \frac{\delta T}{T}
$$

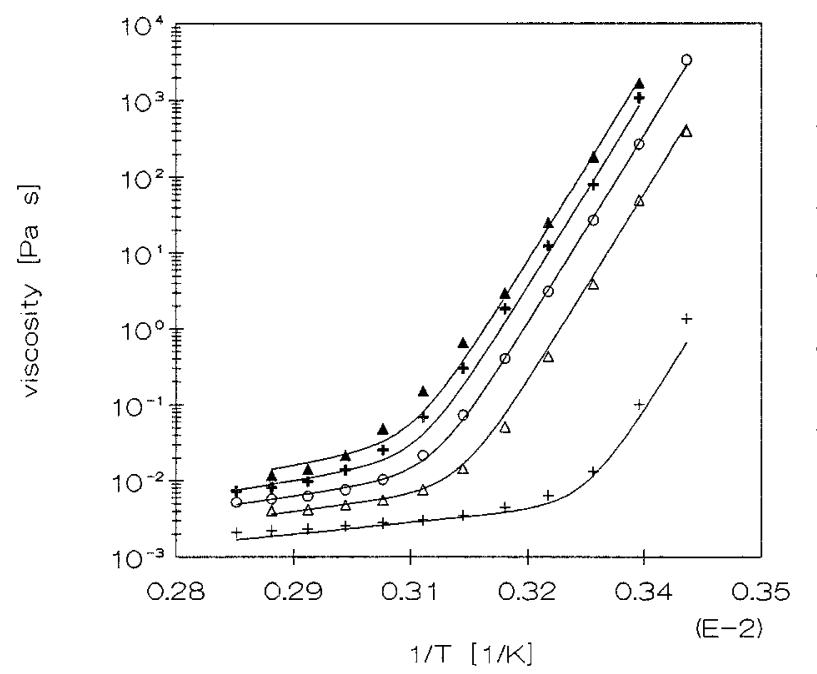

For the parameters, the values given in (15) and (16) can be substituted.

At a temperature of $335 \mathrm{~K}$, and at $c_{\mathrm{pol}}=0.03$, for $\eta_{\text {low }}$, we now find:

$$
\frac{\delta \eta_{\text {low }}}{\eta_{\text {low }}} \simeq 10.0 \frac{\delta T}{T}=10 \frac{0.4}{335} \simeq 0.012 \text {. }
$$

At a temperature of $300 \mathrm{~K}$, for $\eta_{\text {high }}$, we find:

$$
\frac{\delta \eta_{\text {high }}}{\eta_{\text {high }}} \simeq 133 \frac{\delta T}{T}=133 \frac{0.4}{300} \simeq 0.18
$$

As the estimates in (17) and (18) are not the only contributions to the real measurement errors in the viscosities, it can be concluded that a r.m.s. relative deviation of 0.21 , as found in the best-fit curves, does not indicate that relation (14) cannot match the measured viscosity data.

The solid lines in Fig. 6 give the logarithm of $\eta$ according to the expression (14) with the parameter values given in (16).

All parameter values carry with them an uncertainty range. One may ask what the uncertainty range of the fitted parameters is. As outlined above, the accuracy of the viscosity data is not known exactly and, therefore, only an impression of the extent of this range can be given. This was done by varying the "best" parameter values (16) simultaneously in such a way that the parameter sets giving a r.m.s. relative deviation of 0.25 are scanned, thereby registrating their maximum and minimum values. The results are

$$
0.95<\frac{\bar{\eta}}{\bar{\eta}_{\text {best }}}<1.07 ; \quad 0.92<\frac{B}{B_{\text {best }}}<1.17 ;
$$

$$
\begin{aligned}
& \text { + fraction } \\
& 0.015 \\
& \triangle \text { fraction } \\
& 0.03 \\
& \text { fraction } \\
& 0.04 \\
& 0.05
\end{aligned}
$$
0.06

Fig. 6. Zero shear rate solution viscosity as a function of inverse absolute temperature. Symbols: experimental values (same as in Fig. 2). Solid curves: five-parameter fit according to Eq. (14) 


$$
\begin{aligned}
& 0.95<\frac{b}{b_{\text {best }}}<1.07 ; \quad 0.11<\frac{d^{*}}{d_{\text {best }}^{*}}<1.60 ; \\
& 0.41<\frac{\zeta}{\zeta_{\text {best }}}<1.45 .
\end{aligned}
$$

From these results it is concluded that the values of $\bar{\eta}$, $B$, and $b$ are much more accurate than those of $d^{*}$ and $\zeta$.

As was mentioned already, it is inherent in moreparameter fits that the uncertainties in the values of the various parameters are coupled with each other. In the present case the measurements, see Figure 2, on the one hand cover a range of inverse temperature where $\eta_{\text {high }}$ strongly dominates that is large compared to the intermediate range (where both $\eta_{\text {high }}$ and $\eta_{\text {low }}$ contribute significantly to viscosity), and on the other hand the range of $1 / T$ where $\eta_{\text {low }}$ strongly dominates is of the same order as the intermediate range. Therefore, the intermediate range of $1 / T$ plays an important role in the determination of the parameter values of the set $\left\{d^{*}, \zeta\right\}$ and plays only a small role in the determination of the parameter values of the set $\{\bar{\eta}, B, b\}$.

The uncertainty in the values of $d^{*}$ and $\zeta$ is rather large but, due to the coupling mentioned above, the uncertainty in the value of the combined quantity $\left(d^{*}+\zeta / T\right)$, for a certain temperature $T$, is not. This is demonstrated in Table 1 . We will use the results for the combined quantity later on.

Having established the empirical expression for $\eta$, we now must explore the physical background of the relatively simple viscosity behavior.

\section{The basic model}

\section{Linear viscoelasticity}

We consider liquids in the limit of zero shear rate. Then:

$$
\tau=\eta \dot{\gamma}
$$

where $\eta$ is the Newtonian (shear) viscosity, a quantity not depending on shear rate. Its value equals the macroscopic work dissipated in the liquid per unit volume and time, at unit shear rate. This dissipation is that of the work being continuously stored in local molecular configurations and - in the stationary state - being dissipated (i.e., turned into internal energy) at the same rate.

This continuous local "storage" and "dissipation" is, in the limit of zero shear rate, not different from that (as a result of fluctuations) in the liquid when left at rest; only the source of the work is different. Newtonian viscosity, therefore, is a property connected with the dynamics of the molecular structure of the liquid in its state of thermodynamic equilibrium. It can be written as an integral over the time constants characterizing these dynamics. To see this, we recall some results of linear response theory [7], applied to systems subjected to simple shear. The shear stress response on a unit step in shear, imposed at time $t=0$, is called the relaxation function, and denoted by $G\{t\}$. According to theory, $G\{t\}$ may be represented as:

$$
G\{t\}=H\{t\} \int_{0}^{\infty} d \lambda g_{\text {tot }}\{\lambda\} \exp (-\lambda t),
$$

where $H\{t\}$ denotes the Heaviside unit step function, where $1 / \lambda$ has the meaning of a relaxation time $\tau$, and where $\lambda^{2} g_{\text {tot }}\{\lambda\}$, after substitution of $\lambda$ by $1 / \tau$, is called the relaxation time distribution function, denoted by $h\{\tau\}$.

The function $g_{\text {tot }}\{\lambda\}$ may consist of a continuous (index $c$ ) and/or a discrete part, and thus may be written as

$$
g_{\text {tot }}\{\lambda\}=g_{c}\{\lambda\}+\sum_{j=0}^{N} G_{j} \delta\left(\lambda-\lambda_{j}\right) ;
$$

$G_{j}$ is called the relaxation strength of the discrete mechanism $j$, of which there are $(N+1)$ in total. $\delta(\lambda)$ is the Dirac delta distribution.

Table 1. Parameter values leading to almost similar fits

\begin{tabular}{llll}
\hline & First possibility & Second possibility & Third possibility \\
\hline $\bar{\eta}[\mathrm{Pa} \mathrm{s}]$ & $\exp (-119.13)$ & $\exp (-120.26)$ & $\exp (-119.24)$ \\
$B$ & 0.18851 & 0.17412 & 0.19085 \\
$b$ & 39521 & 39773 & 39570 \\
$d^{*}$ & -126.19 & -107.01 & -140.48 \\
$\zeta[\mathrm{K}]$ & 58279 & 51825 & 63090 \\
r.m.S. rel. deviation & 0.2116 & 0.2078 & 0.2125 \\
$d^{*}+\zeta / T$ at $320 \mathrm{~K}$ & 55.9 & 54.9 & 56.7 \\
$d^{*}+\zeta / T$ at $345 \mathrm{~K}$ & 42.7 & 43.2 & 42.4 \\
\hline
\end{tabular}


Substitution of (21) in (20) gives:

$$
\begin{aligned}
G\{t\}= & H\{t\} \int_{0}^{\infty} d \lambda g_{c}\{\lambda\} \exp (-\lambda t) \\
& +H\{t\} \sum_{j=0}^{N} G_{j} \exp \left(-\lambda_{j} t\right) \\
= & H\{t\} \int_{0}^{\infty} d \tau h_{c}\{\tau\} \exp (-t / \tau) \\
& +H\{t\} \sum_{j=0}^{N} G_{j} \exp \left(-t / \tau_{j}\right) .
\end{aligned}
$$

The Fourier transform of $G\{t\}$ equals the complex viscosity $\eta\{i \omega\}$, so from (20) and (22):

$$
\begin{aligned}
\eta\{i \omega\}= & \int_{0}^{\infty} d \lambda \frac{g_{\text {tot }}\{\lambda\}}{\lambda+i \omega}=\int_{0}^{\infty} d \lambda \frac{g_{c}\{\lambda\}}{\lambda+i \omega} \\
& +\sum_{j=0}^{N} G_{j} \frac{1}{\lambda_{j}+i \omega} .
\end{aligned}
$$

Suppose $\lambda_{0}=0$ and $g_{c}\{0\}$. Then (23) can be written as:

$$
\eta\{i \omega\}-\frac{G_{e}}{i \omega}=\int_{0}^{\infty} d \lambda \frac{g_{c}\{\lambda\}}{\lambda+i \omega}+\sum_{j=1}^{N} G_{j} \frac{1}{\lambda_{j}+i \omega},
$$

in which $G_{e}$ denotes the total strength of the mechanisms, if present, for which $\lambda=0$, i.e., for which $\tau=\infty$.

The zero-frequency limit of the real part of the lefthand side of (24) equals the zero-frequency limit of $\eta^{\prime}\{\omega\}$, the real part of $\eta\{i \omega\}$. So, according to (24),

$$
\lim _{\omega \rightarrow 0} \eta^{\prime}\{\omega\}=f_{0}^{\infty} d \lambda \frac{g_{\mathcal{c}}\{\lambda\}}{\lambda}+\sum_{j=1}^{N} G_{j} \frac{1}{\lambda_{j}},
$$

where the bar in the integral symbol indicates that the Cauchy Principal Value of the integral has to be taken.

When a system does contain mechanisms for which $\tau=\infty$, work can be permanently stored in it; a steady shear results in an ever increasing stress, and Eq. (19) does not apply. When there are no such mechanisms, the lefthand side of (25) becomes equal to the Newtonian viscosity, and can, according to (25), be written as:

$$
\begin{aligned}
\eta & =\int_{0}^{\infty} d \lambda \frac{g_{\mathrm{tot}}[\lambda\}}{\lambda}=\int_{0}^{\infty} d \lambda \frac{g_{c}\{\lambda\}}{\lambda}+\sum_{j=1}^{N} G_{j} \frac{1}{\lambda_{j}} \\
& =\int_{0}^{\infty} d \tau \tau h\{\tau\}+\sum_{j=1}^{N} G_{j} \tau_{j} .
\end{aligned}
$$

This is the integral over time-constants meant in the second paragraph of this section; the sum in (26) can be looked at as the result of the integration of the $\delta$ distributions in (21).

The distribution function $g_{\text {tot }}\{\lambda\}$ may consist of contributions from groups of "storage-and-dissipation mechanisms" (we call these relaxation mechanisms) with a different physical nature; each group, when the system is a liquid, gives its own contribution to $\eta$.

A difference in physical nature may bring with it a difference in, e.g., the temperature dependence of the contributions. The empirical relations, arrived at in the preceding section, suggest the latter to be the case in the triblock-copolymer solutions investigated.

\section{Molecular model}

We now turn our attention to polymer solutions. Their viscosity can be written as:

$$
\eta=\eta_{0}+\eta_{\mathrm{pol}}
$$

where $\eta_{0}$ is the viscosity, at the temperature and pressure considered, of the pure solvent. The interest then goes to the measurement and interpretation of $\eta_{\mathrm{pol}}$.

The contribution $\eta_{0}$ stems from mechanisms, not known in detail, in which temporarily stored free energy is energetic as well as entropic, and with which, in common low viscosity solvents, very short relaxation times, say $<10^{-8} \mathrm{~s}$, must be associated because up to frequencies of $10^{8}$ per second or even higher, $\eta^{\prime}\{\omega\}$ of these solvents does not display any frequency dependence.

The mechanisms behind the contribution $\eta_{\text {pol }}$ are understood to be dominantly entropic, and well known theories, based upon bead-and-spring models and reasonably confirmed by experiments, about the contribution $G_{\mathrm{pol}}\{t\}$ to the relaxation function exist [3c], [8]. We now investigate what the beadand-spring model can tell as about $G_{\text {pol }}\{t\}$, and thus about $\eta_{\text {pol }}$, in our triblock-copolymer solutions.

In these solutions, the polymer molecules might be classified according to one or both of their A-ends being "free" or "fixed". We call an end "free" when it has a certain non-zero mobility, and "fixed" when it has a zero mobility with respect to its direct surroundings; in the latter case, it has to move along with those surroundings.

As the only way in which a chain end can be "fixed" we take here its being part of a domain containing several A-ends; domains containing just two 
ends will be supposed to be absent, so for the present discussion we leave out the possibility of free ABA ring-molecules, and that of chains of ABA-molecules linearly connected to each other by domains containing just two A-ends. The molecules we then wish to consider are: $\alpha$-type molecules (both ends are free), $\beta$ type molecules (one end free and the other end fixed), $\gamma$-type molecules (both ends fixed and being part of different domains), $\delta$-type molecules (both ends fixed and being part of one and the same domain).

Modeling all molecules as consisting of $N+1$ "beads" (at which the friction with the surroundings is thought to be localized) connected by $N$ Gaussian springs the relaxation times and strengths of these types can be calculated, e.g., by following a scheme given by Zimm c.s. [9]. The spectrum is discrete, and contains $N+1$ relaxation times $\tau_{p}(p=0,1, \ldots, N)$. There is always one trivial time, say $\tau_{0}$, which is infinite. It corresponds to a purely translational mode in which no work can be stored. The other times, for the free-draining case, and in the limit of infinite dilution, are given by

$$
\left.\begin{array}{l}
\tau_{p}^{\alpha}=a \sin ^{-2}\left(\frac{p \pi}{2(N+1)}\right) \\
\tau_{p}^{\beta}=a \sin ^{-2}\left(\frac{(2 p-1) \pi}{2(2 N+1)}\right) \\
\tau_{p}^{\gamma}=a \sin ^{-2}\left(\frac{(p-1) \pi}{2 N}\right) \\
\tau_{p}^{\delta}=a \sin ^{-2}\left(\frac{(p-1) \pi}{2 N}\right)
\end{array}\right\},
$$

where $p=1, \ldots, N$, and where $a$ is a constant, inversely proportional to $N^{2}$, and approximately proportional to $\eta_{0}$. The result for $\tau_{p}^{\alpha}$ is well known; we did not find the other ones explicitly mentioned in standard literature.

In reality, there is no free draining, by which the spacing of relaxation times is narrower than that according to the expressions (28). Furthermore, as polymer mass fraction increases, the relaxation times, in particular the long ones, become longer and their spacing becomes wider. These restrictions on the results (28) will not, however, affect the essential conclusions of the following reasoning.

Both in the sets $\tau_{p}^{\gamma}$ and $\tau_{p}^{\delta}$ we have:

$$
\tau_{1}=\infty \text {. }
$$

This is a consequence of the zero mobility assigned to both the first and the last bead of those molecules, by which the direct surroundings of these beads dictate to them their position relative to each other. Any of the non-infinite relaxation times corresponds to the motion of polymer molecules according to a certain normal mode; the sets of normal modes are just a little different for $\alpha, \beta, \gamma$, and $\delta$-type molecules.

Now let, at time $t=0$, an instantaneous unit step in shear be imposed on the system. Then all beads will follow the deformation affinely; within the picture of the bead-and-spring model, all work done on the polymer molecules is stored in the springs and, per unit volume, given by $G_{\text {pol }}[t=0]$. Next, this work is dissipated, and stress decays according to $G_{\mathrm{pol}}\{t\}$. In our case the four contributions to $G_{\mathrm{pol}}\{t\}$ are:

$$
\begin{aligned}
& G_{\mathrm{pol}}^{\alpha}\{t\}=\frac{\varrho_{\mathrm{pol}}^{\alpha}}{m_{\mathrm{pol}}} k T\left[\sum_{p=1}^{N} \exp \left(-t / \tau_{p}^{\alpha}\right) H\{t\}\right] \\
& G_{\mathrm{pol}}^{\beta}\{t\}=\frac{\varrho_{\mathrm{pol}}^{\beta}}{m_{\mathrm{pol}}} k T\left[\sum_{p=1}^{N} \exp \left(-t / \tau_{p}^{\beta}\right) H\{t\}\right] \\
& G_{\mathrm{pol}}^{\gamma}\{t\}=\frac{\varrho_{\mathrm{pol}}^{\gamma}}{m_{\mathrm{pol}}} k T\left[1+\sum_{p=2}^{N} \exp \left(-t / \tau_{p}^{\gamma}\right) H\{t\}\right] \\
& G_{\mathrm{pol}}^{\delta}\{t\}=\frac{\varrho_{\mathrm{pol}}^{\delta}}{m_{\mathrm{pol}}} k T\left[\sum_{p=2}^{N} \exp \left(-t / \tau_{p}^{\delta}\right) H\{t\}\right]
\end{aligned}
$$

where $\varrho_{\mathrm{pol}}$ denotes polymer density (mass of polymer per unit volume of solution), $m_{\text {pol }}$ the mass of a polymer molecule, and $k$ Boltzmann's constant. The relaxation strengths are given by the factor in front of that between square brackets.

Only $G_{\text {pol }}^{\gamma}\{t\}$ contains a contribution $\left(\varrho_{\mathrm{pol}}^{\gamma} / m_{\mathrm{pol}}\right) k T$ which does not fade away with time. It finds its origin in the fixation of the end-beads (which fixation leads to a relaxation time $\tau=\infty$ ) of $\gamma$-type molecules in different domains. The position of these domains relative to each other changes with the deformation imposed on the system, and a corresponding amount of work is and remains stored.

In $G_{\mathrm{pol}}^{\delta}\{t\}$ a corresponding contribution, $\left(\varrho_{\mathrm{pol}}^{\delta} / m_{\mathrm{pol}}\right) k T$, is lacking. The end-beads of the $\delta$ type molecules being fixed in one and the same domain, their relative position or, at least, their distance does not change on system deformation. So no work can be stored in the $\tau=\infty$ mechanism of $\delta$-molecules. 
Now suppose a steady shear, with shear rate $\dot{\gamma}$, is imposed on the solution at $t=0$. Then, after transients have faded away, and in the limit of $\dot{\gamma} \rightarrow 0$, the polymer contributes to the work dissipated per unit volume and time by an amount equal to $\dot{\gamma}^{2} \eta_{\mathrm{pol}}^{\prime}\{\omega \rightarrow 0\}$. From (25) and (29), we find

$$
\begin{aligned}
\dot{\gamma}^{2} \eta_{\mathrm{pol}}^{\prime}\{\omega \rightarrow 0\}= & \dot{\gamma}^{2} \frac{k T}{m_{\mathrm{pol}}} \\
& \left(\varrho_{\mathrm{pol}}^{\alpha} \sum_{p=1}^{N} \tau_{p}^{\alpha}+\varrho_{\mathrm{pol}}^{\beta} \sum_{p=1}^{N} \tau_{p}^{\beta}\right. \\
& \left.+\varrho_{\mathrm{pol}}^{\gamma} \sum_{p=2}^{N} \tau_{p}^{\gamma}+\varrho_{\mathrm{pol}}^{\delta} \sum_{p=2}^{N} \tau_{p}^{\delta}\right) .
\end{aligned}
$$

In addition, an ever increasing amount of work, given by

$$
\frac{1}{2} \frac{\varrho_{\mathrm{pol}}^{\gamma}}{m_{\mathrm{pol}}} k T(\dot{\gamma} t)^{2},
$$

is being stored in the system.

To portray the essence of the behavior of a dynamic network, now suppose the constraint on the mobility of at least one of the ends of $\gamma$-type molecules is released at $t=t_{f}$. Then the "elastic" energy given by (31), is dissipated in relaxation processes associated with $\beta$-type molecules. Now suppose $t_{f} \gg \tau_{1}^{\beta}$, and suppose very soon after relaxation a number of polymer molecules corresponding to $\varrho_{\mathrm{pol}}^{\gamma}$ again turn into $\gamma$ type molecules, are released at $t=2 t_{f}$, etc. Then all the time, in addition to the amount given by (30), an amount of work given by:

$$
\frac{1}{2} \frac{\varrho_{\mathrm{pol}}^{\gamma}}{m_{\mathrm{pol}}} k T \dot{\gamma}^{2} t_{f}
$$

is dissipated per unit volume and time. In the solution, which now behaves as a liquid, this leads to a contribution, $\eta_{1}^{\gamma}$, given by

$$
\eta_{1}^{\gamma}=\frac{1}{2} \frac{\varrho_{\mathrm{pol}}^{\gamma}}{m_{\mathrm{pol}}} k T t_{f}
$$

to the viscosity of the solution. Comparing this result with (26), we write it as

$$
\eta_{1}^{\gamma}=G_{1}^{\gamma} t_{f}
$$

A result very similar to (32) was already obtained by Lodge [10] (transient network model). In the
Lodge model, however, $\eta_{1}^{\gamma}$ comes out twice as high, when $t_{f}$ is set equal to $\bar{t}_{\text {res }}$, the average time a chain end is part of one and the same domain, in the Lodge model. The reason is that in the Lodge model there is a continuous storage and dissipation of free energy, whereas in the fictitious saw-tooth model considered above the free energy stored is dissipated after fixed time intervals. From now on we replace (32) by

$$
\eta_{\mathrm{l}}^{\gamma}=\frac{\varrho_{\mathrm{pol}}^{\gamma}}{m_{\mathrm{pol}}} k T \bar{t}_{\mathrm{res}} .
$$

Whereas the non-infinite times of the sets $\tau_{p}^{\alpha}, \tau_{p}^{\beta}, \tau_{p}^{\gamma}$, and $\tau_{p}^{\delta}$ are related to mobility and elasticity properties of polymer chains in a solvent, the time constant $\bar{t}_{\text {res }}$ is of a different physical nature: it is related to the average time an A-end is part of one and the same domain.

The amount of stored work given by (31) equals that of a permanent network, containing, per unit volume, $\varrho_{\mathrm{pol}}^{\gamma} / m_{\mathrm{pol}}$ Gaussian polymer chains between crosslinks, and which has been subjected to a shear $\gamma$ with a value equal to $\dot{\gamma} t$ in (31) (after which crosslinkpositions have been fixed ${ }^{1)}$ at positions corresponding with affine deformation). For this reason the contribution $\eta_{1}^{\gamma}$, given by (33), will be called $\eta_{\text {network }}$. So

$$
\eta_{\text {network }}=\frac{\varrho_{\text {pol }}^{\gamma}}{m_{\text {pol }}} k T \bar{t}_{\mathrm{res}} .
$$

The remaining polymer contribution to viscosity is given by the righthand side of (30) divided by $\dot{\gamma}^{2}$, and we call it $\eta_{\text {pol, free }}$.

Then $\eta_{\text {pol }}$, the total polymer contribution to viscosity, becomes

$$
\eta_{\mathrm{pol}}=\eta_{\mathrm{pol}, \text { free }}+\eta_{\text {network }} .
$$

Introduction of (35) into (27) gives

$$
\eta=\eta_{0}+\eta_{\text {pol, free }}+\eta_{\text {network }} .
$$

The result (36) is an example of a specific decomposition of (26) into contributions to viscosity from groups of relaxation mechanisms with a different physical nature.

1) Without this fixation, $Q_{\mathrm{pol}}^{\gamma} / m_{\mathrm{pol}}$ should be replaced by $\left(\varrho_{\mathrm{pol}}^{\gamma} / m_{\mathrm{pol}}-v\right)$, where $v$ is the number of crosslinks per unit volume [11-13]; the quantity $v k T \gamma^{2} / 2$ is the amount of work which is not being stored when there is no (artificial) constraint on crosslink positions upon deformation. 
The presence of the contribution $\eta_{\text {network }}$ stems from that of molecules with their ends fixed in different domains. The result $\tau_{1}^{\gamma}=\infty$, to which this leads, does not depend on the conditions, temperature and polymer mass fraction, which do affect the values of the non-infinite relaxation times of the system.

As for the latter effect, temperature changes $\eta_{0}$ and, by that, the non-infinite relaxation times of the polymer. Together with polymer mass fraction, it may also affect the distribution of polymer molecules over the various types $(\alpha, \beta, \gamma, \delta)$ considered. According to the results (28), however, there is not much difference between the relaxation times of the sets $\alpha, \beta, \gamma$, and $\delta$, and we may write [see (30)] in good approximation $^{2)}$ :

$$
\eta_{\mathrm{pol}, \text { free }}=\eta_{\mathrm{pol}}^{\prime}\{\omega \rightarrow 0\} \simeq \frac{k T}{m_{\mathrm{pol}}} \varrho_{\mathrm{pol}} \sum_{p=1}^{N} \tau_{p}^{\alpha} .
$$

This means that $\eta_{\text {pol, free }}$ is hardly sensitive for the distribution of polymer molecules over the various types, and that its value is about the same as that of $\eta_{\text {pol }}$ of a comparable solution of non-associating polymer molecules.

The conclusion (37) is based upon the non-infinite relaxation times of the sets $\alpha$, etc., as given in (30), being approximately the same. There is no reason why this will be very different for the sets of the true relaxation times (containing the influence of hydrodynamic interaction and polymer mass fraction). So we write

$$
\eta_{\text {pol, free }} \simeq \eta_{\text {pol. non ass. }},
$$

where $\eta_{\text {pol.non ass. }}$ is the polymer contribution to the viscosity of an "equivalent" (same polymer ${ }^{3)}$, same solvent, same mass fraction, same temperature) solution of non-associating polymer molecules.

One subtle point (about the quantitative correctness of (38) at low temperatures) will be made in the discussion.

For further use, we write

\footnotetext{
2) Provided the amount of $\beta$-type molecules (their longest relaxation time is about four times as large as $\tau_{1}^{\alpha}, \tau_{2}^{\gamma}$, and $\tau_{2}^{\delta}$ ) is and remains relatively small, which we suppose to be the case.

3) As such, one might just take polyisoprene with a backbone length equal to that of the ABA-molecules; remember that the stretched length of the B-block is about 40 times that of an A-block.
}

$$
\eta_{0}+\eta_{\text {pol, free }} \equiv \eta_{\text {free }},
$$

by which (36) becomes

$$
\eta=\eta_{\text {free }}+\eta_{\text {network }}
$$

\section{Comparison of theory with experiment}

The empirical expression (14) was found to describe quite well the temperature and mass fraction dependence of the zero-shear-rate viscosity of our system. We now investigate to which extent the empirical decomposition of $\eta$ into two contributions, $\eta_{\text {low }}$ and $\eta_{\text {high }}$, is covered by the theory developed in the preceding section.

According to this theory, the viscosity (see (36)) can, indeed, be decomposed into separate contributions, and it was argued that the contribution $\eta_{\mathrm{pol}, \text { free }}$ would not differ much from the polymer contribution to the viscosity of an equivalent ${ }^{4)}$ solution of non-associating polymer molecules. This implies that $\eta_{\text {free }}$, defined in (39), would be the viscosity of such a system.

It is interesting, therefore, to inspect the empirical quantity $\eta_{\text {low }}$ more closely. Introducing into (12) the values of $\left(d^{*}+\zeta / T\right)$ taken from Table 1, middle column, we find,

$$
\text { (at } \begin{aligned}
T & =320 \mathrm{~K}) \\
\eta_{\mathrm{low}} & =\eta_{0}\{T=320 \mathrm{~K}\} \exp \left(54.9 c_{\mathrm{pol}}\right),
\end{aligned}
$$

(at $T=345 \mathrm{~K}$ )

$$
\eta_{\text {low }}=\eta_{0}\{T=345 \mathrm{~K}\} \exp \left(43.2 c_{\mathrm{pol}}\right) \text {. }
$$

Taylor expansion of the exponent in (41 a) gives

$$
\begin{aligned}
\eta_{\text {low }}= & \eta_{0}\{T=320 \mathrm{~K}\} \\
& \cdot\left(1+54.9 c_{\mathrm{pol}}+\frac{1}{2}(54.9)^{2} c_{\mathrm{pol}}^{2}+\ldots\right) .
\end{aligned}
$$

A more usual expansion is that in $\varrho_{\text {pol }}$, the polymer density (mass of polymer per unit volume of solution). The relation between $c_{\mathrm{pol}}$ and $\varrho_{\mathrm{pol}}$ reads

$$
c_{\mathrm{pol}}=\frac{\varrho_{\mathrm{pol}}}{\varrho},
$$

where $\varrho$ is the density of the solution. Introducing (43)

\footnotetext{
4) See preceding footnote.
} 
in (42) and assigning to $\varrho$ the value $756 \mathrm{kgm}^{-3}$, the density of $n$-tetradecane at $320 \mathrm{~K}$, we obtain

$$
\begin{aligned}
\eta_{\text {low }}= & \eta_{0}\{T\} \\
& \cdot\left(1+0.0726 \varrho_{\mathrm{pol}}+\frac{1}{2}(0.0726)^{2} \varrho_{\mathrm{pol}}^{2}+\ldots\right) .
\end{aligned}
$$

For polymer solutions it is usual to write

$$
\eta=\eta_{0}\{T]\left(1+[\eta] \varrho_{\mathrm{pol}}+k_{H}[\eta]^{2} \varrho_{\mathrm{pol}}^{2}+\ldots\right)
$$

where $[\eta]$ is the intrinsic viscosity (limiting viscosity number) and $k_{H}$ the Huggins constant. When $\eta_{\text {low }}$ would be the viscosity of a polymer solution, then (see (44) and (45)) its intrinsic viscosity and Huggins constant would be

$$
[\eta]=0.073 \mathrm{~m}^{3} \mathrm{~kg}^{-1}=73 \mathrm{ml} \mathrm{g}^{-1} ; k_{H}=0.5
$$

When using (41 b), we would have found:

$$
[\eta] \approx 0.057 \mathrm{~m}^{3} \mathrm{~kg}^{-1}=57 \mathrm{ml} \mathrm{g}^{-1}, \quad k_{H}=0.5 \text {. }
$$

These are reasonable values for common polymer solutions [14].

The exponent in the empirical expression (12) automatically leads to the value 0.5 for the Huggins constant. Experimental data about $\eta_{\text {low }}$, however, do not allow for an expression containing more than two parameters, and the exponent in (12) quite well covers the overall behavior, including that at rather high mass fractions.

The main conclusion is that the empirical contribution $\eta_{\text {low }}$ does not differ significantly from the viscosity of an equivalent solution of non-associating polymer; the viscosity of such a solution was indicated by $\eta_{\text {pol. non ass. }}$.

Consequently,

$$
\eta_{\text {low }} \simeq \eta_{0}+\eta_{\text {pol. non ass }} .
$$

In view of (39), we conclude

$$
\eta_{\text {free }} \simeq \eta_{\text {low }} .
$$

Combination of (13), (40), and (46) gives

$$
\eta_{\text {network }} \simeq \eta_{\text {high }}
$$

Introducing (34) for $\eta_{\text {network }}$ and (5) for $\eta_{\text {high }}$, we find

$$
\frac{\varrho_{\mathrm{pol}}^{\gamma}}{m_{\mathrm{pol}}} k T \vec{t}_{\mathrm{res}} \simeq \eta_{\text {high }}=\bar{\eta} c_{\mathrm{pol}} \exp \left(-\frac{B}{c_{\mathrm{pol}}}\right) \exp \left(\frac{b}{T}\right)
$$

Table 2. Values of $\left(\varrho_{\mathrm{pol}}^{\gamma} / \varrho_{\mathrm{pol}}\right) \bar{t}_{\mathrm{res}}[\mathrm{s}]$ according to (49) for some $\left\{c_{\text {pol }}, T\right\}$ combinations -

\begin{tabular}{llll}
\hline & $c_{\mathrm{pol}}=0.015$ & $c_{\mathrm{pol}}=0.030$ & $c_{\mathrm{pol}}=0.060$ \\
\hline$T=293 \mathrm{~K}$ & $4.2 \cdot 10^{-3}$ & 1.4 & $2.5 \cdot 10^{1}$ \\
$T=303 \mathrm{~K}$ & $4.6 \cdot 10^{-5}$ & $1.5 \cdot 10^{-2}$ & $2.8 \cdot 10^{-1}$ \\
\hline
\end{tabular}

or, using (43),

$$
\begin{aligned}
\frac{\varrho_{\mathrm{pol}}^{\gamma}}{\varrho_{\mathrm{pol}}} \bar{t}_{\mathrm{res}} & =\frac{m_{\mathrm{pol}}}{k T \varrho} \eta_{\mathrm{high}} \\
& =\frac{m_{\mathrm{pol}}}{k T \varrho} \bar{\eta} \exp \left(-\frac{B}{c_{\mathrm{pol}}}\right) \exp \left(\frac{b}{T}\right) .
\end{aligned}
$$

With $\varrho \simeq 756 \mathrm{~kg} \mathrm{~m}^{-3}$ and $m_{\mathrm{pol}} \approx 2.7 \cdot 10^{-19} \mathrm{~kg}$, and using for $\bar{\eta}, B$, and $b$ the values given in (16), we find for $\left(\varrho_{\mathrm{pol}}^{\gamma} / \varrho_{\mathrm{pol}}\right) \bar{t}_{\mathrm{res}}$ the values given in Table 2 .

Because $\varrho_{\mathrm{pol}}^{\gamma} / \varrho_{\mathrm{pol}} \leqslant 1, \bar{t}_{\mathrm{res}}$ is never smaller than these values, provided (49) is correct.

For a further analysis we need the separate values of $\bar{t}_{\mathrm{res}}$, or $\varrho_{\mathrm{pol}}^{\gamma} / \varrho_{\mathrm{pol}}$. Such data will be presented in another paper [2]. Here, we just mention some preliminary results on $\bar{t}_{\text {res }}$ obtained by Smits and Bousché [15] from early dynamic mechanical measurements. They found, at $c_{\mathrm{pol}}=0.015, \bar{t}_{\mathrm{res}}$ values of, roughly, $10,1.5$, and $0.1 \mathrm{~s}$ at $T=289,295$, and $299 K$, respectively. That is,

$$
\left.\frac{\partial}{\partial T}{ }^{10} \log \left(\frac{\bar{t}_{\mathrm{res}}}{t_{0}}\right)\right|_{c_{\mathrm{pol}}} \simeq-0.2 \mathrm{~K}^{-1},
$$

where $t_{0}$ is some arbitrarily chosen reference value of $\bar{t}_{\mathrm{res}}$.

From (49) and the temperature dependence of $\eta_{\text {high }}$, we find:

$$
\left.\frac{\partial}{\partial T}{ }^{10} \log \left(\frac{\bar{t}_{\mathrm{res}}}{t_{0}}\right)\right|_{c_{\mathrm{pol}}} \simeq-0.19 \mathrm{~K}^{-1}, \text { at } T=300 \mathrm{~K} \text {, }
$$

in which the value of $b$ mentioned in (16) has been used (the contribution $-\partial^{10} \log (T) / \partial T$ is negligible).

There are reasons why the " $\simeq$ "-sign in (49) might have to be replaced by a "<"-sign; these will be indicated in the discussion. As for temperature dependence, however, the relation (49) does not seem to be in conflict with reality.

In the Lodge transient network model, the segments are considered to leave the network according to a mono-molecular decay process. Doing the same for the $A$-ends leaving an $A$-domain in our system, we write 


$$
\frac{d n_{A}}{d t}=-h n_{A}
$$

where $n_{A}$ is the number of $A$-ends present in domains, and $d n_{A} / d t$ the change in that number, per unit time, due to chain ends leaving domains. It can easily be shown that $h$, the reaction constant, equals $1 / \bar{t}_{\text {res }}$, the inverse of the average time a chain end is part of one and the same domain.

For $h$, we may write

$$
h \sim \exp \left(-\frac{\Delta \mu_{0}}{k T}\right) \text {, }
$$

where $\Delta \mu_{0}$ is the difference between the standard chemical potential of a molecule in the (highest) activated state and that of one with an $A$-end in a domain. On writing

$$
\Delta \mu_{0}=\Delta h_{0}-T \Delta s_{0},
$$

where $\Delta h_{0}$ and $\Delta s_{0}$ are the differences in the standard enthalpy and the standard entropy in the two states, and on assuming that $\Delta s_{0}$ and $\Delta h_{0}$ themselves do not depend on temperature, we obtain

$$
\begin{aligned}
& h \sim \exp \left(-\frac{\Delta h_{0}}{k T}\right), \\
& \bar{t}_{\text {res }}=h^{-1} \sim \exp \left(+\frac{\Delta h_{0}}{k T}\right) .
\end{aligned}
$$

In view of the almost equal temperature dependence of $\log \left(\bar{t}_{\text {res }}\right)$ and $\log \left(\eta_{\text {high }}\right)$, noticed above, the parameter $b$ in the expression for $\eta_{\text {high }}$ might give an indication about $\Delta h_{0}$, called the enthalpy of activation. From the value of $b$ given in (16), we arrive at

$$
\Delta h_{0}=5.6 \cdot 10^{-19}[\mathrm{~J}] \text {. }
$$

At $T=300 \mathrm{~K}$ this corresponds to about 130 times $k T$.

\section{Gelation temperature and gelation curve}

In thermoreversibly gelating systems, like the ones studied here, it is arbitrary at which temperature the system, known to develop a "plateau" in the storage modulus as temperature, say, decreases, should be said to change from a liquid into a gel. The viscosity behavior of our ABA solutions suggests a simple proposal. It was found that the decomposition (13) of the viscosity $\eta$ into a contribution $\eta_{\text {low }}$, described by (12), and a contribution $\eta_{\text {high }}$, described by (5), quite well represents the dependence of solution viscosity on temperature and polymer mass fraction. At constant $c_{\text {pol }}, \eta_{\text {low }}>\eta_{\text {high }}$ at high temperatures, and the reverse is found at low temperatures. We propose to call the temperature at which $\eta_{\text {high }}$ equals $\eta_{\text {low }}$ the gelation temperature $\left(T_{\mathrm{gel}}\right)$, that is,

$$
\text { (at } \left.T_{\text {gel }}\right) \quad \eta_{\text {high }}=\eta_{\text {low }} \text {, }
$$

or, using (5), (12), and (9),

$$
\begin{aligned}
& \tilde{\eta} c_{\mathrm{pol}} \exp \left(\frac{-B}{c_{\mathrm{pol}}}\right) \exp \left(\frac{b}{T_{\mathrm{gel}}}\right)=j\{0\} \exp \left(\frac{D\{0\}}{T_{\mathrm{gel}}}\right) \\
& \quad \times \exp \left(\left(d^{*}+\frac{\zeta}{T_{\mathrm{gel}}}\right) c_{\mathrm{pol}}\right) .
\end{aligned}
$$

The equality (51) implies the following relation between $T_{\text {gel }}$ and $c_{\text {pol }}$ :

$$
T_{\mathrm{gel}}=\frac{b-D\{0\}-\zeta c_{\mathrm{pol}}}{d^{*} c_{\mathrm{pol}}+\frac{B}{c_{\mathrm{pol}}}+\ln \left(\frac{j\{0\}}{\bar{\eta} c_{\mathrm{pol}}}\right)} .
$$

The plot of $T_{\text {gel }}$ vs $c_{\text {pol }}$ may be called the gelation curve. The gelation curve that follows from (52), with the parameter values given in (16), is shown in Fig. 7.

The definition (50) of the gelation temperature may also be applied separately to the viscosity data for each sample with a certain polymer mass fraction. For

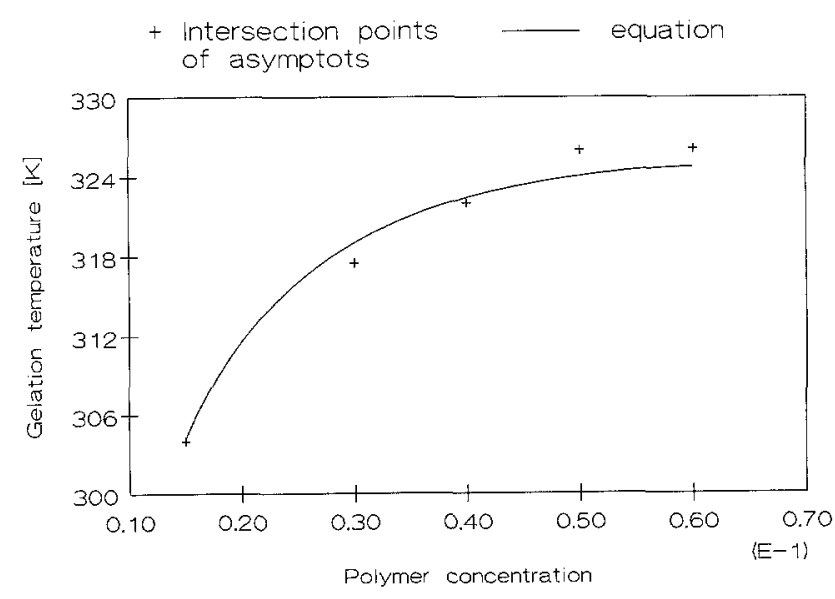

Fig. 7. Gelation temperature as a function of polymer mass fraction. Symbols: intersection points of the asymptotes (straight lines $(\bar{a}+\bar{b} / T)$ and $(\bar{c}+\bar{d} / T)$, with $\bar{a}, \bar{b}, \bar{c}$, and $\bar{d}$ depending on mass fraction) in Fig. 2. Solid curve: gelation temperature according to Eq. (52) 
$\eta_{\text {high }}$ and $\eta_{\text {low }}$, instead of (5) and (12), we then adopt the expression

$$
\eta_{\text {high }}=\exp \left(\bar{a}+\frac{\bar{b}}{T}\right) ; \quad \eta_{\text {low }}=\exp \left(\bar{c}+\frac{\bar{d}}{T}\right)
$$

in which $\bar{a}, \bar{b}, \bar{c}$, and $\bar{d}$ are functions of mass fraction which have not to be specified. This means that $\exp (\bar{a})$ may differ from $g\left(c_{\text {pol }}\right\}$ given by (4) and (16), $\bar{b}$ from $b$ given in (16), $\exp (\bar{c})$ from $\left(j\{0\} \exp \left(d^{*} c_{\mathrm{pol}}\right)\right)$ given by (15) and (16), and $\bar{d}$ from $\left(D\{0\}+\zeta c_{\mathrm{pol}}\right)$ given by (15) and (16).

According to (50), $T_{\text {gel }}$ is given by

$$
T_{\text {gel }}=\frac{\bar{b}-\bar{d}}{\bar{c}-\bar{a}} .
$$

For $\eta$, we again tried (13), that is,

$$
\eta=\eta_{\text {high }}+\eta_{\text {low }}
$$

but this time by introducing the expressions (53) into it; that is,

$$
\eta=\exp \left(\bar{a}+\frac{\bar{b}}{T}\right)+\exp \left(\bar{c}+\frac{\bar{d}}{T}\right) .
$$

Curve fits of (55) to the viscosity data for each mass fraction separately led to five sets of best values of $\bar{a}, \bar{b}, \bar{c}$, and $\bar{d}$. When introduced into (55) this results in the dashed lines in Fig. 2. When introduced into (54) this results in the $T_{\text {gel }}$ values indicated by symbols in Fig. 7. As to be expected, these temperatures do not deviate much from the gelation curve calculated from (52).

It can easily be checked that $T_{\text {gel }}$, as defined in (50), happens to coincide with the temperature at which the second derivative of $\ln (\eta)$ to $1 / T$, at constant $c_{\text {pol }}$, goes through its maximum; this is true for $\eta$ described by (55) and also, therefore, for $\eta$ described by (14). The proposal (50) is a special case of a recent, more general proposal [16].

\section{Discussion}

The present work is part of a more comprehensive study into the properties of ABA-triblock copolymer solutions in a very poor solvent for $A$. The zero-shear rate viscosity was measured and analyzed.

Empirically, we found (see Eqs. (13) and (14)) that the solution viscosity was quite well described by a sum of two contributions, each one depending in its own way on temperature and polymer mass fraction.

From a molecular picture of the system, we arrived at the expression (36); the terms $\eta_{\text {pol, free }}$ and $\eta_{\text {network }}$ were (see (37) and (34)) specified contributions to the righthand side of (26), the general expression for the zero-shear rate viscosity of a liquid.

It was argued that $\eta_{\text {pol, free }}$ is about equal to the polymer contribution to the viscosity of an equivalent solution of non-associating polymer; this was expressed by (38). Finally, after stating that $\eta_{\text {low }}$ behaves as to be expected from a solution of non-associating polymer, the term $\eta_{\text {high }}$ could be identified (see (46) and (47)) as $\eta_{\text {network}}$.

We now discuss the validity of (38) at low temperatures, around and below $T_{\text {gel }}$, introduced before. There the time $\bar{t}_{\text {res }}$ is becoming increasingly long and $A$-domains, though not containing the same $A$-ends all the time, are becoming relatively sluggish objects. They may start acting as obstacles for those polymer molecule motions to which the relaxation times $\tau_{p}^{\alpha}(p=1, \ldots, N), \tau_{p}^{\beta}(p=1, \ldots, N), \tau_{p}^{\gamma}(p=2, \ldots, N)$, and $\tau_{p}^{\delta}(p=2, \ldots, N)$ are related. In particular, the longer times of these sets will be affected (made longer), and these contribute most to viscosity. In addition, the cage-like structures (local or long-range (temporary) networks), to be expected when a sufficient number of molecules is $\gamma$-type, may slow down the polymer molecule motions, again leading to an increase of the (long) relaxation times and, thus, of the viscosity-contribution $\eta_{\text {pol. free }}$.

The conclusion is that, in the low-temperature region, $\eta_{\text {pol. free }}$ is probably larger than $\eta_{\text {pol. non ass. }}$ defined after (38). So we replace (38) by

$$
\eta_{\text {pol. free }}>\eta_{\text {pol. non ass. }}
$$

Accordingly, the " $\simeq$ "-sign in (46) should be replaced by a " $>$ "-sign, and the " $\simeq$ "-signs in (47), (48), and (49) by "<"-signs.

As we were not able to predict these effects quantitatively from theory, we tried to determine these by experiment. For this purpose, we added pure $B$ (polyisoprene) to a solution of ABA in $n$-tetradecane, and intended to measure the zero shear viscosity somewhat below $T_{\text {gel }}$. The mixture, however, prepared somewhat above $T_{\text {gel }}$, displayed phase separation on cooling. So the experiment we had in view could not be carried out. Apparently, the domain-and/or cage-formation, becoming important on cooling, imposes restrictions on the conformations of the polymer molecules. The $B$-molecules among 
these, having no affinity to $A$-domains, separate from an ABA-containing region for entropic reasons.

When obstacles (domains, cages) impose restrictions upon polymer conformations, they will certainly also reduce the mobility of parts of polymer molecules and, thereby, increase their relaxation times. A quantitative relation is still lacking.

As for the influence of (dissolved) polymer molecules on each other's mobilities and, thus, on relaxation times, strong effects have been predicted and observed [17], especially in the semi-dilute and concentrated regime. Calculations for the dilute and concentrated regime were performed by, among others, Geurts and Wiegel [18]. By choosing permanent constraints instead of randomly appearing and disappearing ones, the presence of (sluggish) domains may be simulated, and their influence might be studied.

It might also be informative to measure the viscosity of homopolymer solutions to which rigid colloidal particles for which the polymer has no affinity have been added. The possibility to do so depends on whether such systems, too, do display phase separation, as it is predicted and observed [18], or do not.

In conclusion, we can say that the viscosity behavior, reported and analyzed in this paper, can be given a theoretical background, although some interesting problems remain to be solved. The definition of the gelation temperature (see (50)) is based upon the empirical behavior and has no relation with these problems.

\section{Acknowledgement}

Thanks are due to Y. de Fluiter, for pointing out to us the results $(28)$.

\section{References}

1. Möller M, Mühleisen E, Omeis J (1988) In: Burchard W, Ross-Murphy SB (eds) Physical Networks. Elsevier Applied Science, London [1 a]: pp 45-64; [1 b]: pp $1-14$
2. Spitteler PHJ (paper in preparation)

3. Ferry JD (1980) In: Viscoelastic Properties of Polymers, Third Edition, John Wiley and Sons, New York [3a]: Chapter 10; [3b]: Chapter 11; [3c]: Chapter 9

4. Winter HH, Chambon F (1968) J Rheol 30(2):367 - 382

5. Chambon F, Winter HH (1987) J Rheol 31(5):683-697

6. Press WH, Flannery BP, Teukolsky SA, Vetterling WT (1986) In: Numerical Recipes, The art of scientific computing, First Edition, Cambridge University Press, Cambridge, Chapter 10

7. Gross B (1953) In: Mathematical Structure of the Theories of Viscoelasticity, First Edition, Hermann, Paris

8. Bird RB, Curtiss CF, Armstrong RC, Hassager $O$ (1987) In: Dynamics of Polymeric Liquids, Second Edition, John Wiley and Sons, New York, Volume 2, Chapter 15

9. Zimm BH, Kilb RW (1959) J Pol Sci 37:19-41

10. Lodge AS (1956) Trans Faraday Soc 52:120-130

11. Duiser JA, Staverman AJ (1965) In: Prins JA (ed) Physics of Noncrystalline Solids, North Holland Publ Comp, Amsterdam, pp 376-387

12. Graessley WW (1975) Macromolecules 8:186-190

13. Staverman AJ (1982) Adv Polym Sci 44:73 - 101

14. Morawetz H (1965) In: Macromolecules in Solution, Interscience Publ, New York, Chapter 6

15. Smits HJE, Bousché O (1986) Measurements performed at this laboratory

16. Visscher K, Mijnlieff PF (1990) Makromol Chem, Macromol Symp 39:99-123

17. Doi M, Edwards SF (1986) In: The Theory of Polymer Dynamics, Clarendon Press, Oxford

18. Geurts JW, Wiegel FW (1987) Mod Phys Letters B1 $1: 57-60$

19. De Hek H, Vrij A (1981) J Coll Interf Science $84: 409-422$

(Received February 7, 1991; in revised form August 12, 1991; accepted September 3, 1991)

Correspondence to:

K. Visscher Rheology Group Dept. of Applied Physics

Twente University, P.O. Box 217

7500 AE Enschede

The Netherlands 